\title{
Nuevos actores en la politica local: un análisis comparativo de su comportamiento en las ciudades de Medellín y Bogotá (1988- 2015) ${ }^{1}$
}

\section{New actors in local politics: a comparative analysis of their behavior in the cities of Medellin and Bogotá (1988-2015)}

\section{Juan Carlos Escobar Escobar iD}

Universidad de Antioquia

juan.escobare@udea.edu.co

\section{Resumen}

El propósito de este artículo es examinar la aparición de nuevos actores de la politica en las ciudades de Bogotá y Medellín entre 1988 y 2015, a partir de la revisión de la bibliografía existente y del análisis de los datos electorales oficiales para Alcaldía y Concejo. El artículo muestra procesos políticos similares y, a su vez, procesos con marcadas diferencias. La más importante de estas es que, en la disputa por la Alcaldia de Bogotá, el dominio electoral de las opciones no bipartidistas, y claramente de izquierda desde el año 2003 hasta el 2015, no sería amenazado por las fuerzas partidistas tradicionales. En Medellín, en cambio, los tradicionales mantuvieron altas posibilidades de retorno, que de hecho se concretaron en algunos eventos electorales posteriores a la aparición de nuevas fuerzas políticas exitosas.

Palabras clave: Partidos políticos, elecciones locales, nuevos actores políticos, democracia, participación política.

\begin{abstract}
The main purpose of this article is to analyze the appearance of new political actors in the cities of Bogota and Medellin between 1998 and 2015, based on the bibliographic inspection. This review includes the results on the ballot boxes, the discursive language and the analysis of the electoral data of Municipal and Town Council. The paper shows similar political processes and deep differences. The most important of these, shows the political party forces would no threatened the domain of the non-two-party options and clearly biased to the left since 2003 and 2015 for the Bogotá Government. On the other hand, in Medellin the traditional parties maintain high possibilities to return, that in fact obtain good results in some of the electoral events after the creation of new successful political forces.
\end{abstract}

Keywords: Political parties, local elections, new political actors, democracy, political participation.

Articulo: Recibido el 9 de septiembre de 2018 y aprobado el 13 de junio de 2019.

\section{Como citar este articulo:}

Escobar Escobar, J. (2019). Nuevos actores en la política local: un análisis comparativo de su comportamiento en las ciudades de Medellín y Bogotá (1988-2015). Reflexión Política 21(42), pp. 177-188. doi: 10.29375/01240781.3363

$1 \quad$ El artículo es producto de mi actividad como investigador principal del proyecto Nuevos partidos políticos: un análisis comparativo de su comportamiento en las ciudades de Medellín y Bogotá (1991-2011), que se llevó a cabo entre marzo de 2017 y marzo de 2018, y contó con recursos del Sistema Universitario de Investigación (SUI) de la Universidad de Antioquia. Dicho proyecto desarrolló además un objetivo de mi tesis de Doctorado en Ciencias Humanas y Sociales de la Universidad Nacional-Sede Medellín, siendo el artículo un requisito para el título de Doctor. Agradezco el apoyo de Juan Camilo Parra Restrepo, quien estuvo en el proyecto en calidad de Joven Investigador Universidad de Antioquia. 


\section{Introducción}

A lo largo de las dos décadas pasadas, los colombianos asisten una y otra vez a la anunciada defunción de los partidos políticos a expensas de los llamados independientes, de los "nuevos", de las terceras fuerzas y de lo que la Registraduría Nacional del Estado Civil denominó como "otras opciones". Las reformas institucionales de la década de los ochenta y principios de los noventa, la incursión en el escenario político de un partido de izquierda como la Unión Patriótica y la retórica de la democracia participativa que intentaba instalarse, generaron la sensación de que algo empezaba a cambiar. Más adelante, el entusiasmo que produjeron los resultados de la Alianza Democrática M-19 en las elecciones para conformar la Asamblea Nacional Constituyente en 1990 y en las elecciones parlamentarias de 1991, hizo pensar a algunos que se estaba ante el quiebre definitivo del sistema político tradicional, o por lo menos ante la reorganización del sistema de partidos que implicaba la muerte del bipartidismo. Incluso, uno de los objetivos explícitos de la Constituyente era el fortalecimiento de los partidos y la ampliación del abanico de posibilidades político-electorales y, quizás con ello, la reconfiguración del sistema de partidos.

Como se sabe, el cambio político y la reorganización del sistema de partidos debieron esperar unas décadas más para concretarse. Las elecciones parlamentarias de 1994 trajeron consigo el reposicionamiento del bipartidismo y la postergación, una vez más, de la consolidación de una opción política que amenazara la permanencia del bipartidismo colombiano. En este, como en otros casos, el marco y el discurso constitucional fueron instrumentalizados por los actores tradicionales, que aprendieron más rápido que el resto las reglas para reposicionarse en el espectro político. Adicionalmente, la criminalización de la política, que implicó el exterminio de la Unión Patriótica y de líderes de otros grupos políticos de izquierda, contribuyó a que el entramado político siguiera dominado por los partidos tradicionales.

Sin embargo, la postergación del quiebre del bipartidismo en el ámbito nacional no fue obstáculo para que el discurso de los independientes, de los sin partido y, en algunos casos, de los antipartido, se extendiera por el país y se instalara especialmente en las grandes ciudades. Todo esto pasaba mientras en algunos países vecinos como Ecuador y Perú se experimentaban, en unos casos incluso desde los años ochenta, procesos de cambio agenciados por políticos o movimientos que no guardaban relación con los actores tradicionales de la política.

El propósito de este artículo es examinar la aparición de nuevos actores de la política en las ciudades de Bogotá y Medellín, a partir de la revisión de la bibliografía existente y de los datos electorales para Alcaldía y Concejo. Para ello, el texto está dividido en dos partes: en la primera se explicitan algunos elementos teóricos y metodológicos que se referencian a lo largo del texto, y en la segunda se abordan los casos concretos de Bogotá y Medellín para ejemplificar la forma en que estos escenarios, de maneras distintas, presenciaron la aparición de nuevos actores de la política que confrontaron el tradicional sistema de partidos.

\section{Elementos teóricos y metodológicos}

\subsection{Antipartidismo, partidos antisistema, outsiders, Break-in parties}

Desde hace varias décadas se han vuelto recurrentes en el lenguaje de la ciencia política términos como declive, desafección o malestar con la política y sus actores. En particular, con el tema de los partidos políticos se pueden identificar, al menos, dos formas de acercarse al fenómeno referido.

Un primer grupo se ha preocupado más por las actitudes de los ciudadanos hacia los partidos, sin tocar mucho el asunto del declive en el apoyo público de los mismos, ya que dirige su mirada a cuestiones como la evolución de la identificación partidista, la participación electoral, entre otros. Tratados desde el tema del antipartidismo (Gidengil, Blais, Neviette y Nadeau, 2001) o los sentimientos antipartidistas (Torcal, Montero y Günther, 2007), estos trabajos asumen que el "sentimiento antipartidista forma parte de una crítica generalizada a otras instituciones democráticas, entre las que se incluirian el parlamento y en términos más generales las élites políticas o los políticos" (Torcal et al., 2007, p. 246).

REFLEXIÓN POLÍTICAAÑO 21 № 42 AGOSTO DE 2019 ISSN (en línea) 2590-8669; IEP - UNAB (COLOMBIA) 
Sin embargo, el centro de estos trabajos se dirige a lo que sienten los ciudadanos por los partidos y a las actitudes de reacción o pasividad que este sentimiento genera.

Otro grupo concentra su análisis tanto en las estructuras organizativas, las funciones y los miembros de los partidos, como en sus actuaciones en el gobierno y en las instituciones representativas. En tal sentido, se han formulado cinco grupos de críticas. Primero, se argumenta que los partidos no reflejan las divisiones más importantes de la sociedad y que, incluso, tienden a ocultarlas al utilizar estratégicamente discursos generales sobre problemas que pueden interesar a una amplia gama de electores. Segundo, estas organizaciones son desbordadas por problemáticas como el medio ambiente, las discusiones sobre el género o la multiculturalidad, asuntos cruciales en la sociedad actual. Tercero, se resalta la ausencia de propuestas programáticas de los partidos, carencia que es suplida por medio de estrategias publicitarias en las que lo fundamental es la imagen de los candidatos, lo que acentúa las tendencias al personalismo. Cuarto, se juzga que los partidos políticos no poseen una organización democrática y que, por el contrario, sus estructuras se caracterizan por su verticalidad y la escasa participación de las bases. Por último, se indica que los partidos hoy cumplen más la tarea de ser voceros del Estado frente a la sociedad civil, que de representar los intereses de esta ante aquel.

El efecto de estos niveles crecientes de malestar con los partidos y con los políticos ha sido el surgimiento de diversos discursos alrededor de los cuales se articulan propuestas electorales y de gobierno, que intentan capitalizar el malestar y promover nuevos modos de hacer política, en los que el candidato presenta como su mejor característica el estar en contra, por fuera, o más allá de los partidos y la clase política tradicional. Estos fenómenos han sido llamados en la literatura politológica de distintas formas: partidos antisistema, outsiders, Break-in parties, entre otros, los cuales se registran en las democracias occidentales.

El concepto de partido antisistema pretendía brindar una solución parcial a algunos problemas analíticos de las formas de oposición, enfocándose no solo en la ideología de los partidos, sino en su éxito para socavar la legitimidad del régimen al cual se opone en su impacto deslegitimador (Sartori, 1976, pp. 132-133). El partido antisistema es concebido por autores como Keren (2000) como un partido que habla en nombre de un imaginario en lugar de la sociedad civil real. Esta distinción (partidos enraizados en imaginarios versus la sociedad civil real) se basa en gran parte en la noción de Comunidades imaginadas de Benedict Anderson (1991), y provee, según Keren, alternativas a algunos de los problemas previamente asociados con la categoría antisistema (Keren, 2000, p. 109).

$\mathrm{El}$ intento del autor es interesante en la medida que busca una definición positiva del partido antisistema, como una organización que procura un estándar de perfección derivado de su compromiso con un imaginario en lugar de la sociedad civil real. Sin embargo, no parece tener un carácter operacional o, por lo menos, tiende a quedar corto a la hora de definir nuevos partidos políticos que no necesariamente están compitiendo contra el sistema.

Otro término bastante recurrente para aludir a la situación descrita es el de outsider. Algunos autores acuden a la dicotomía outsiderinsider para explicar el fenómeno (Barr, 2009, p. 32). La novedad no es aquí requisito de la condición de outsider. Lo que importa, en cambio, es la ubicación del político o del partido en relación al sistema de partidos. Desde esta distinción casi etimológica, el status de outsider depende estrictamente de dicha ubicación, no de la retórica ni de la estrategia. Autores como Lynch (1999) insisten en el discurso condenatorio de la política y los políticos que introduce el outsider, al señalarlos como la causa de los males de las democracias representativas.

Otro grupo de autores se interesa más por la viabilidad, la forma en que irrumpen en el sistema y el éxito o fracaso que allí alcanzan los nuevos partidos. El fracaso o el éxito partidario es realmente una definición polisémica y arbitraria dentro de la discusión teórica sobre los partidos políticos (Harmel y Robertson, 1985). Sin embargo, autores como Lago y Martínez (2010) siguen usando estas categorías u otras que remarcan la idea de la viabilidad de los nuevos partidos, la cual está dada por la forma en que las reglas de juego electoral permiten o no la existencia de nuevas opciones y cómo estas aprenden a jugar dentro de aquellas. Más recientemente se ha usado el término Break-in parties con un interés muy similar: medir el éxito o fracaso de los nuevos partidos que irrumpen en contextos como el de América Latina (Kestler, Krause y Lucca, 2013). 
En Colombia, los trabajos sobre los fenómenos mencionados se han encontrado con la dificultad de clasificar a los nuevos políticos colombianos como outsiders, antisistema o antipolíticos. Esa dificultad se basa en que, efectivamente, los nuevos políticos colombianos que han incursionado y ocupado el poder local (o pugnado por el nacional) suelen ser menos radicales que sus homólogos de los países vecinos. Sin embargo, alguna literatura se ha encargado de estos políticos y partidos emergentes, generalmente como un tema subsidiario de estudio sobre el sistema de partidos y el sistema electoral colombiano (Mainwaring y Scully, 1995; Boudon, 2000; Leal, 1988; Leal y Zamosc, 1990; Gutiérrez, 2006 y 2007).

\subsection{Breve aclaración metodológica}

A pesar de que, como lo indica el título, el periodo en el que se centra el artículo va desde 1988 hasta 2015 -por ser los años de los que se pudo obtener mayor información y escenificar los más importantes cambios políticos-, para las elecciones a Concejo se tienen datos desde 1972 hasta $2011^{2}$, lo que permite tener antecedentes importantes del comportamiento partidista previo a la Elección Popular de Alcaldes. Para el caso de la Alcaldía se toma desde 1988 (por ser esta la primera elección popular de alcaldes del país) hasta 2015. En términos metodológicos generales, es preciso señalar que para la construcción de las gráficas que se muestran en el texto, se usaron datos de la Base de Datos Electorales CEDE, de la Universidad de los Andes, así como los datos de la Registraduría Nacional del Estado Civil.

Además, fue necesario complementar los datos del CEDE con los extraídos de la Registraduría puesto que en algunos casos había información aglomerada bajo la categoría "resto de votación". Así, para los años 1988 y 1990 solo se presenta al candidato ganador, mientras que el resto de la votación está en esa categoría que es necesario desagregar. También es preciso señalar, por motivos metodológicos, que el total de votos se calculó a partir de la sumatoria de las votaciones obtenidas por todos los candidatos más el voto en blanco -los votos nulos no son tenidos en cuenta pues no expresan una opción política partidista-. Finalmente, para los años en que los partidos tradicionales no obtienen votación, dado que no presentaron candidatos, se ingresa a la base de datos el valor 0 .

Para estudiar el comportamiento de los partidos se realizó una clasificación de estos de acuerdo con dos momentos del sistema electoral y de partidos colombianos, usando como punto de partida la reforma política electoral del año 2003. De este modo, antes de 2003 los partidos se clasificaron a través de las categorías Partido Liberal, Partido Conservador, facción ${ }^{3}$ liberal, facción conservadora y otros partidos y coaliciones. Ahora, para efectos posteriores al 2003, luego de la reforma política de ese año y tras el influjo del uribismo en el reordenamiento del sistema de partidos colombianos, dicha clasificación se convierte en tradicionales (Partido Liberal y Partido Conservador), transicionales ${ }^{4}$, otros partidos y nuevos partidos ${ }^{5}$ políticos. En lo que sigue, se realiza una descripción y análisis de las elecciones para Alcaldía y Concejo en cada ciudad, lo anterior con el propósito de mostrar en ellas la aparición paulatina de opciones distintas al voto tradicional y los patrones de voto en cada ciudad.

Como se mencionó en la introducción, los cambios en el nivel local se experimentaron de manera más temprana, aunque también de forma desigual en una y otra ciudad, dejando ver transformaciones en los sistemas de partidos de ambas ciudades y la aparición, inicialmente en ese nivel, de personajes que venían de sectores distintos a la política tradicional, especialmente de la academia.

$2 \quad$ Para las elecciones de Concejo, la información de las bases de datos consultadas no está actualizada hasta 2015 Por ello se trabaja hasta 2011

3 Gutiérrez (2017) entiende, de forma operativa, el término facción como una corriente que se manifiesta en cualquier nivel territorial (nacional, departamental o municipal), la cual se reconoce por un nombre y por un grupo de distintivos, y que desarrolla alguna forma de acción colectiva dentro de un partido. Esto significa que Gutiérrez toma a la facción como un "segundo apellido", como por ejemplo, liberal lopista o arredondista, y conservador ospinista o directorista.

$4 \quad$ Tradicionales que se presentan como nuevos (Gutiérrez, 2007).

$5 \quad$ Nuevos pura sangre que ganaron posiciones en los ejecutivos locales (Gutiérrez, 2007). 


\section{Procesos de cambio político en Bogotá y Medellin}

Una mirada a los estudios realizados y a la dinámica electoral de las ciudades de Medellín y Bogotá deja ver efectivamente cambios importantes, aunque, por supuesto, las generalizaciones son allí menos posibles.

\subsection{Cambios en la política partidista local}

\subsubsection{Bogotá}

Para el caso de Bogotá, desde los primeros años de la década del noventa se advierten algunas tendencias de cambio importantes en el sistema de partidos y en la política local. En efecto, aunque todavía no se habla de un quiebre definitivo del sistema de partidos, se enuncian los indicios que condujeron al mismo, analizando tres hipótesis que sintetizan ese momento de transición: las preferencias electorales no eran para entonces consistentes ni siquiera en el nivel local, los partidos tradicionales todavía eran fuertes (sobre todo el Liberal), pero experimentaban "fatiga de material" (escasez de cuadros y de personal político) y existía una desagregación parcial del bipartidismo (sobre todo en clases medias y altas) que favoreció la importante aparición del voto crítico/cívico (Gutiérrez, 1995).

En una línea muy similar y profundizando una de las hipótesis señaladas, García (2002) sugie- re que en Bogotá desde mediados de los años noventa aparecen sistemas de partidos paralelos, que funcionan de forma diferente en los distintos eventos electorales locales. Esta hipótesis es apoyada con la idea de que el sistema de partidos bogotano estaba experimentando un proceso de desinstitucionalización. Esto se percibe, dice García (2002), en aspectos como la variación del Número Efectivo de Partidos (NEP), los altos niveles de volatilidad electoral, el personalismo alarmante del ejercicio político y la pérdida de identidades partidistas.

La idea más o menos generalizada, fundamentada por supuesto en los estudios revisados y en la percepción de operadores políticos específicos (Guzmán, 2005), es que la década del noventa representa un antes y un después tanto en la política como en el sistema político de esta ciudad; seguramente con las características y las particularidades de cada evento electoral (no es lo mismo votar por alcalde que por concejal), pero siempre con la existencia de movimientos y políticos con bases electorales estables que hicieron imposible, incluso hasta hoy, el retorno de un partido político con marbete tradicional a la Alcaldía.

Los resultados son evidentes: los partidos tradicionales y las otras figuras (los otros, los nuevos y las coaliciones) tienen una relación inversamente proporcional, pues en la medida en que uno aumenta sus resultados electorales, el otro los disminuye. Es claro que entre 1988 y 1992 hay una participación

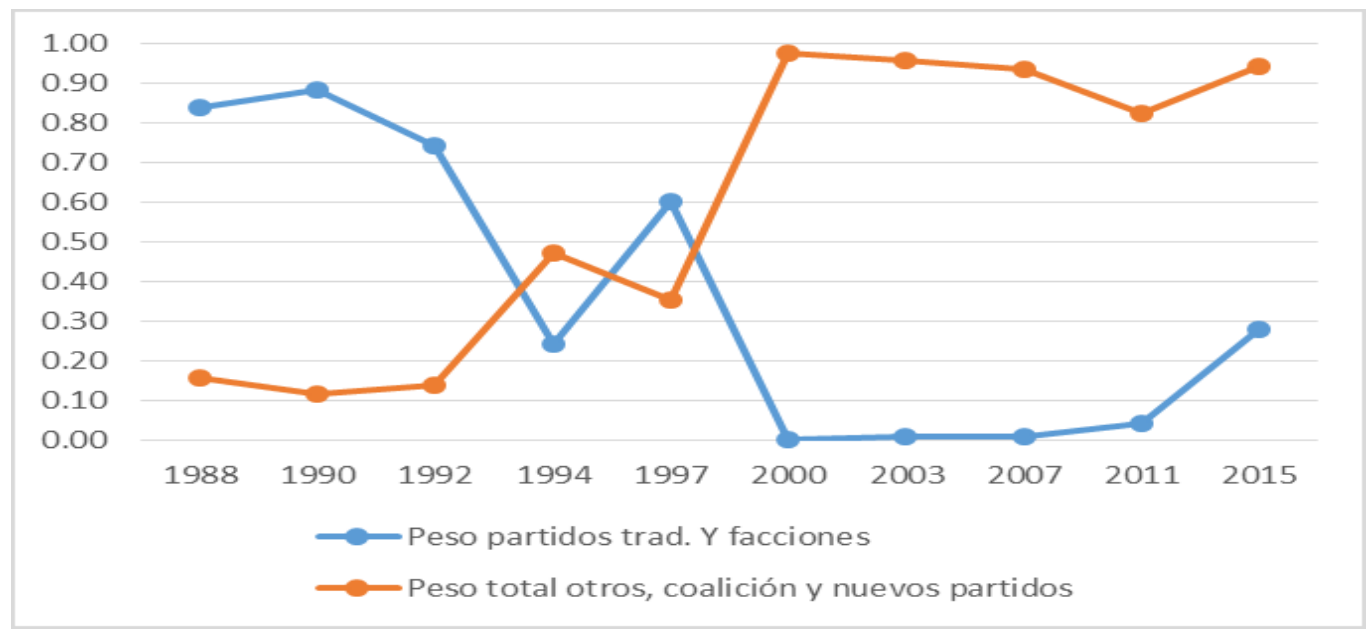

Figura 1. Peso $^{6}$ de partidos tradicionales versus nuevos partidos, otros y coaliciones. Alcaldía de Bogotá (1988-2015).

Fuente: Construcción propia con datos tomados de la Base de Datos Electorales CEDE (Universidad de los Andes) y de la Registraduría Nacional del Estado Civil.

$6 \quad$ El Índice de peso o Tasa de participación por peso se define como el total de la votación obtenida por un partido, movimiento político o candidato sobre el total de votos válidos. Es decir, cuánto pesa cada partido, movimiento o candidato en relación con la votación válida de la ciudad en una circunscripción determinada y en una elección dada. 
minoritaria de estas figuras que emergen como fuerza política en 1994, y continúan con una importante participación en 1997, para finalmente obtener la hegemonía a partir del año 2000. Se podría hablar de tres momentos de este periodo:

- Primer momento (1988-1992): en el año 1988, de 13 candidatos de las figuras diferentes a los tradicionales -es decir, los otros partidos y las coaliciones- resaltan 3 , puesto que las otras 10 votaciones son casi insignificantes. Así, se tiene en cuenta a María Eugenia Rojas (creó una coalición que rememoraba la Anapo), Clara López Obregón (Unión Patriótica) y Carmelina Wilches (Movimiento Metapolítico), quienes obtienen el $10,90 \%, 3,62 \%$ y $1,11 \%$ del peso de la votación total en dichas elecciones. Para las elecciones de 1990, de 8 candidatos de las figuras diferentes, resaltan de nuevo 3, y por las mismas razones. Estos son Carlos Pizarro (Alianza Democrática M-19), Carlos Romero (Unión Patriótica) y Jesús Antonio Castro (Movimiento Metapolítico), quienes logran el 7,79\%, 2,47\% y 1,15\% de la votación, respectivamente. Finalmente, en las elecciones de 1992 se cuenta nuevamente con 13 candidatos de las otras opciones, de los cuales resalta Carlos Alonso Lucio López (M-19), Gerardo Ardila Serrano (M-19), Jaime Turriago Caicedo (Unión Patriótica) y Jorge Enrique Orozco (Movimiento Metapolítico). Estos candidatos obtienen 4,83\%, 2,87\%, 1,95\% y 1,57\%. De este modo, es perceptible cómo en este periodo de tiempo hay una incipiente votación por las figuras distintas a los partidos tradicionales.

- Segundo momento (1994-1997): este es un momento de ruptura importante en la política bogotana. En 1994, por las figuras distintas se presenta el candidato Antanas Mockus, quien gana con un peso del $64,51 \%$ de los votos. Es decir, obtiene el doble que el candidato oficial del Partido Liberal, Enrique Peñalosa, quien obtiene 30,11\%, y del candidato conservador Carlos Moreno de Caro, con 2,68\%. Para el año 1997, momento en que se da el fraccionamiento del Partido Liberal y gana Peñalosa Londoño, esta vez por una facción del mencionado partido, se presentan 6 candidatos por las otras figuras diferentes a los partidos tradicionales. Así, son perceptibles Carlos Moreno de Caro y Juan Carlos Flórez Arcila, quienes obtienen el $31,32 \%$ y $3,05 \%$ de los votos, respectivamente.
- Tercer momento (2000-2015): en este momento se consolida el voto no tradicional en Bogotá, especialmente el voto de izquierda. Durante este periodo todos los alcaldes elegidos en esta ciudad hacen parte de las figuras diferentes a los partidos políticos tradicionales. Excepto por Enrique Peñalosa y Antanas Mockus, el resto de los elegidos eran candidatos de partidos de izquierda con gran peso en esta ciudad.

Si bien hay una dificultad para precisar los verdaderos "independientes" de aquellos que no lo son (lo que con el tiempo fue incluso más sencillo y posible), esto no guarda relación directa con la persistencia de la lógica bipartidista ni con la posibilidad, por esa vía, del retorno de esquema bipartidista al gobierno de Bogotá con el argumento de las oleadas cíclicas (Barreto, 2010, p.76) que pudieron ser importantes en los noventa (Gutiérrez, 1995).

No obstante, las lógicas tradicionales pueden estar presentes, y de hecho lo siguen estando en la política de esta ciudad si se miran los resultados para Concejo que parecen ratificar la existencia, hasta hace muy pocos años, de sistemas de partidos paralelos.

En la Figura 2 se advierte la persistencia, hasta las elecciones de 2000, de un voto muy tradicional en el Concejo capitalino, pese a que los cambios en la disputa por la Alcaldía comienzan a evidenciarse desde antes. En este año, las fuerzas tradicionales y las otras expresiones políticas obtienen casi la misma votación; en los tres siguientes eventos electorales ya no se ve un Concejo tan marcadamente tradicional, sobre todo por la llegada de fuerzas transicionales (Cambio Radical entre ellos) que empiezan a posicionarse quitándole curules a liberales y a conservadores.

\subsubsection{Medellin}

El caso de Medellín difiere en algunos aspectos importantes del bogotano. Sobre el sistema de partidos tradicionales y sus transformaciones pueden leerse dos perspectivas de análisis. Reina (2008) ha enfocado su atención en la dinámica político-electoral de la ciudad de Medellín. El autor centra su análisis en Compromiso Ciudadano, movimiento que ha hecho presencia en la ciudad de Medellín desde la contienda electoral del año 2000, y que ocupó el primer cargo municipal en 2003-2007 y 2008-2011, rompiendo así con el marcado bipartidismo existente en la ciudad. En 


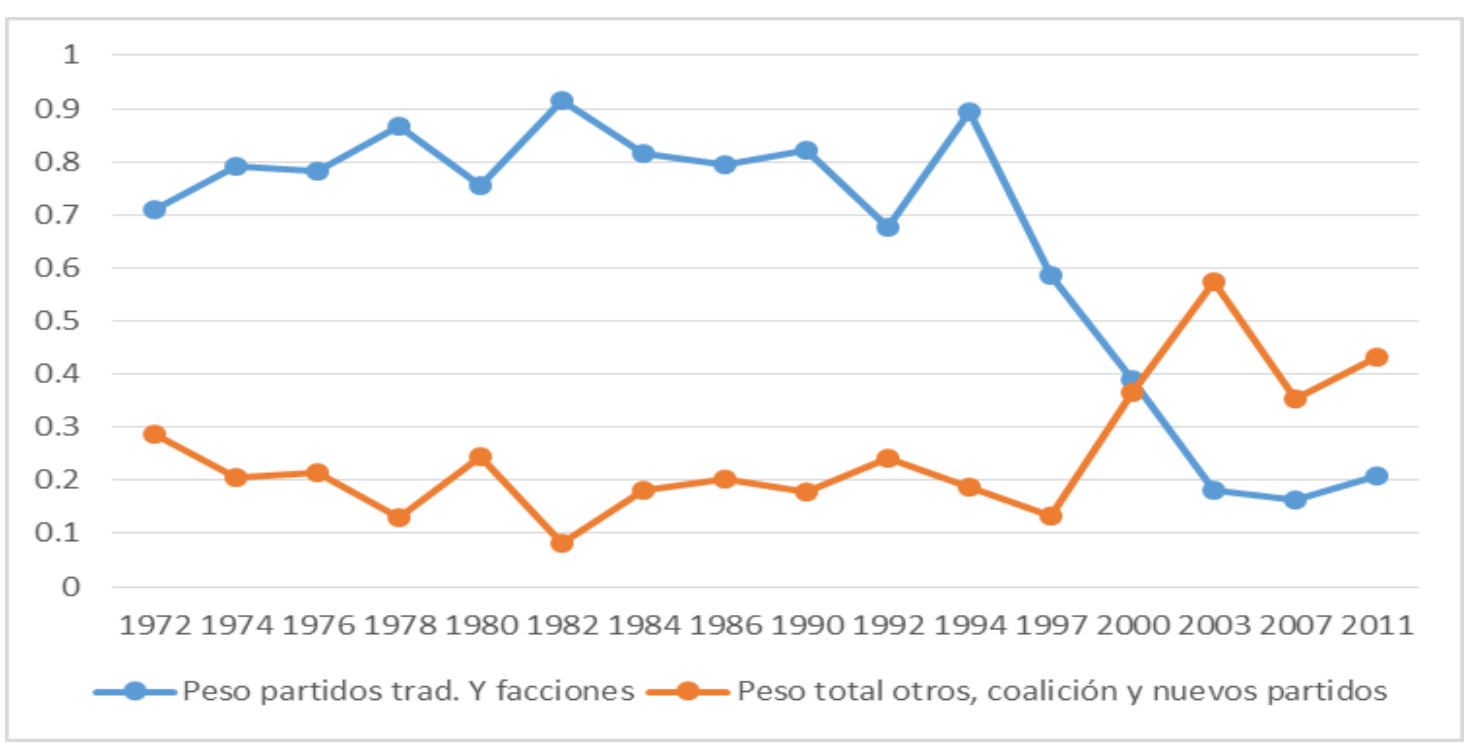

Figura 2. Peso de partidos tradicionales versus nuevos partidos, otros y coaliciones. Concejo de Bogotá (1988-2015).

Construcción propia con datos tomados de la Base de Datos Electorales CEDE (Universidad de los Andes) y de la Registraduría Nacional del Estado Civil.

cuanto a la victoria obtenida por Sergio Fajardo en el año 2003, catalogada como un golpe de opinión generado principalmente por el cansancio hacia el bipartidismo, el autor detalla una consistencia electoral, en algunas zonas de la ciudad, del movimiento Compromiso Ciudadano, desde su surgimiento en las contiendas electorales en el año 2000 con nichos de votación específicos.

La investigación de Reina (2008) se presenta como un interesante ejercicio en donde se analiza la ruptura del bipartidismo en la ciudad de Medellín en las contiendas electorales para la Alcaldía, empleando como elementos de argumentación y demostración índices socio-económicos y la evolución del comportamiento electoral en la ciudad.

Por su parte, el texto de Arenas y Escobar (2012) pretende mostrar de qué manera la década de los noventa se constituyó en un período importante para entender el posterior reacomodamiento del sistema de partidos en lo local. Para tal propósito, se plantea un análisis de las estrategias organizativas con fines electorales de las expresiones locales de los partidos Liberal y Conservador, de los niveles de fraccionamiento alcanzados por ambas colectividades en este período y de la dinámica electoral de la ciudad. Las continuidades y cambios que vivió la ciudad permiten entender la permanencia en aquella década de los tradicionales, pero, igualmente, la aparición dispersa y fragmentada de alternativas al bipartidismo que solo tendrán juego político en la década posterior, hasta ocupar el primer cargo político de la ciudad.

Los resultados de las nuevas expresiones de la política comienzan a ser considerables cuando en el año 2000, con el aval del Partido Movimiento Alianza Social Indígena (ASI) (aunque su grupo político desde entonces se llamó Compromiso Ciudadano), Sergio Fajardo Valderrama obtiene el tercer lugar en las elecciones para alcalde con el 15,88\% de la votación, un resultado más favorable que la segunda facción conservadora que participó en dichas elecciones y que obtuvo la mitad de los votos que el candidato ganador. En las siguientes elecciones, en el año 2003, Sergio Fajardo es elegido alcalde de Medellín a través del mismo partido y con una votación del 48,75\% respecto al total de votos válidos.

Las elecciones de 2007 continúan esta misma tendencia de disminución de votos de los partidos tradicionales, pues en dichas elecciones el Partido Liberal no presenta candidatos, y el candidato del Partido Conservador, Gabriel Jaime Rico, apenas obtiene el 2,64\% de los votos. Así, para estas elecciones hay una amplia gama de candidatos que se presentan a través de opciones diferentes a los partidos tradicionales, aunque algunos de estos candidatos tienen un pasado reciente innegable en la política tradicional de la ciudad. Por ejemplo, por 


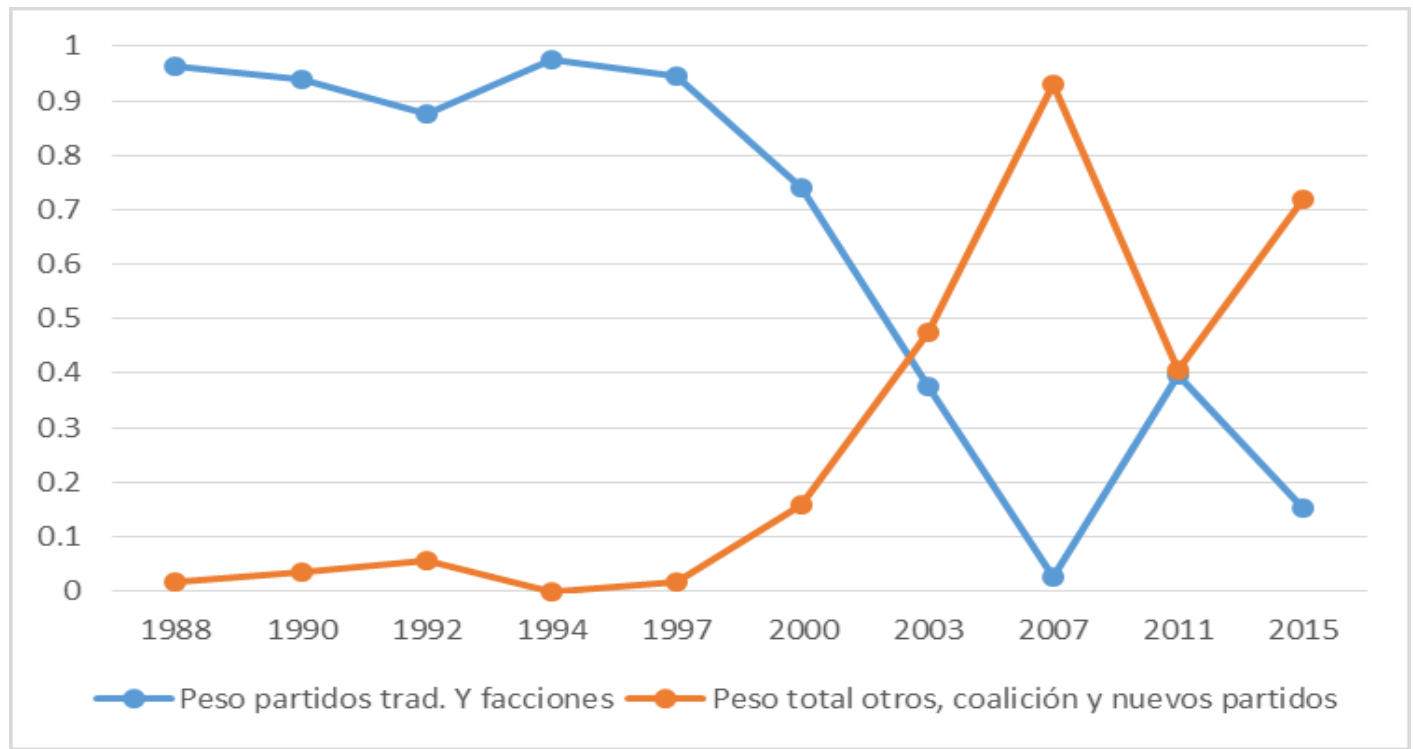

Figura 3. Peso partidos tradicionales vs peso otros partidos, nuevos partidos y coaliciones. Alcaldía de Medellín (19882015).

Fuente. Construcción propia con datos tomados de la Base de Datos Electorales CEDE (Universidad de los Andes) y de la Registraduría Nacional del Estado Civil.

el Partido Verde, se postula Sergio Naranjo, en la que sería su cuarta aspiración a la Alcaldía ${ }^{7}$; por el Polo Democrático Alternativo, Luis Guillermo Pardo Cardona; y por el Partido Autoridades Indígenas de Colombia (AICO), Carlos Ignacio Cuervo Valencia. Estos obtienen el 1,3\%, 2,4\% 0,9\%, respectivamente. En cuanto a los movimientos, clasificados como otros partidos, participa el exalcalde Luis Pérez, con el movimiento Todos con Luis Pérez, que obtiene el $37 \%$ de la votación y Jhon Bazurto Gil, que con el movimiento $A$ lo bien obtiene el 0,4\%. En definitiva, a través de estas elecciones se consolida la caída de los partidos tradicionales, en tanto las votaciones por las otras figuras agrupan el 92,89\% de los votos.

Sin embargo, dicha tendencia es interrumpida para las elecciones de 2011. En ellas se presentan candidatos a través de figuras diferentes a los políticos tradicionales como Luis Fernando Muñoz Ramírez (Polo Democrático Alternativo) y Jacqueline Tolosa Castaño (Movimiento Independiente de Renovación Absoluta), quienes logran el $1,6 \%$ y $2 \%$ de la votación, respectivamente. A los anteriores se suma la nueva participación del exalcalde Luis Pérez, quien esta vez intenta llegar a la Alcaldía a través del movimiento Firmes Por Medellín, quedando en segundo lugar nuevamente y logrando el 36,76\% de los votos. Si bien los resultados de estos 3 candidatos significan el 40,43\% de la votación, hay varios hechos significativos.

En primer lugar, la votación de estas formas nuevas y distintas a los partidos tradicionales descendió del 92,89\% al 40,43\%. En segundo lugar, resalta la presencia de Federico Gutiérrez, quien a través de un partido transicional (Partido de la U) ganó el $19,92 \%$ de los votos. En tercer lugar, el candidato Aníbal Gaviria Correa, perteneciente al Partido Liberal (aunque haciendo parte de una coalición donde confluían igualmente sectores importantes de movimientos no tradicionales), ganó las elecciones con el 39,64\% de los votos. Así, a pesar de que hay una significativa participación de esas otras formas, la Alcaldía para ese año retorna a las manos de un partido tradicional que encabezaba la coalición ganadora.

Finalmente, en las elecciones de 2015 el comportamiento político de los partidos retorna a la tendencia que tenía antes de las elecciones de 2011. Para estas elecciones, el candidato del Partido Liberal, Eugenio Prieto, renuncia, y el candidato del Partido Conservador, el repitente Gabriel Jaime Rico, obtiene el 16,29\% de los votos. No hay participación de los

$7 \quad$ El Índice de peso o Tasa de participación por peso se define como el total de la votación obtenida por un partido, movimiento político o candidato sobre el total de votos válidos. Es decir, cuánto pesa cada partido, movimiento o candidato en relación con la votación válida de la ciudad en una circunscripción determinada y en una elección dada. 
partidos transicionales, y las otras formas agrupan el $77,12 \%$ de las votaciones. Así, gana Federico Gutiérrez con el movimiento Creemos (35,89\%), mientras que Héctor Manuel Hoyos Meneses, por el Polo Democrático Alternativo, obtiene el 1,3\%. El exalcalde Alonso Salazar, por el Partido Alianza Verde, logra el 5,4\%, y Juan Carlos Vélez Uribe, del recién creado partido Centro Democrático, queda en segundo lugar con el 34,49\%.

En síntesis, no se puede mostrar en Medellín momentos claramente diferenciados de la evolución del sistema de partidos, como sí pudo observarse en el caso de Bogotá. Arenas y Escobar (2012) sostienen que, si bien se dio una suerte de reordenamiento del sistema de partidos local, los partidos tradicionales continúan teniendo una parte importante de los escaños del Concejo y amplias posibilidades de disputar el poder ejecutivo local.

En la Figura 4 se muestra cómo el comportamiento electoral de las figuras distintas a los partidos tradicionales y sus facciones, es decir, los otros, los nuevos partidos y las coaliciones no tradicionales, no supera, a excepción de una vez, el 30\% de los votos. Es perceptible, entonces, que a partir de 1990 hay un aumento de la votación de dichas figuras, que pasan de votaciones poco significativas a acercarse y mantenerse alrededor del $20 \%$.

Además, es necesario dejar presente que en las elecciones de 2007 y 2011, cuando la votación de los tradicionales es muy similar a la votación por las figuras distintas, el nuevo factor que emerge es la votación de los partidos transicionales. En el 2007 la participación de estos es del 32,97\%, mientras que en 2011 llega a 36,81\%. Resaltan en ambos periodos el Partido Cambio Radical y el Partido de la U.

Las diferencias en la manera en que se dio el cambio político electoral en ambas ciudades Bogotá y Medellín- son evidentes. Primero porque la transformación en Medellín se presentó de manera tardía, comparado con lo que había pasado una década atrás en la capital y en otras ciudades grandes e intermedias. Segundo, porque los tradicionales en Medellín siguen teniendo juego y posibilidades reales de ganar el poder local, como lo demostraron los comicios de 2007 y 2011 , mientras en Bogotá dejaron de tener peso electoral en la disputa por la Alcaldía. Finalmente, es interesante señalar una importante coincidencia: en ambas ciudades las dinámicas electorales para Alcaldía difieren de las de Concejo, como lo dejan ver algunos estudios (García, 2002; Arenas y Escobar, 2000; Pizano, 2002; Gutiérrez, 2007).

\subsection{Antipoliticos, civicos, independientes y algo más}

Desde los años ochenta y con mucha más fuerza a partir del proceso que condujo a la Constitución Política de 1991, el lenguaje político de la época estuvo dominado por las alusiones a lo cívico, lo independiente y lo antipolítico. Para entonces, la

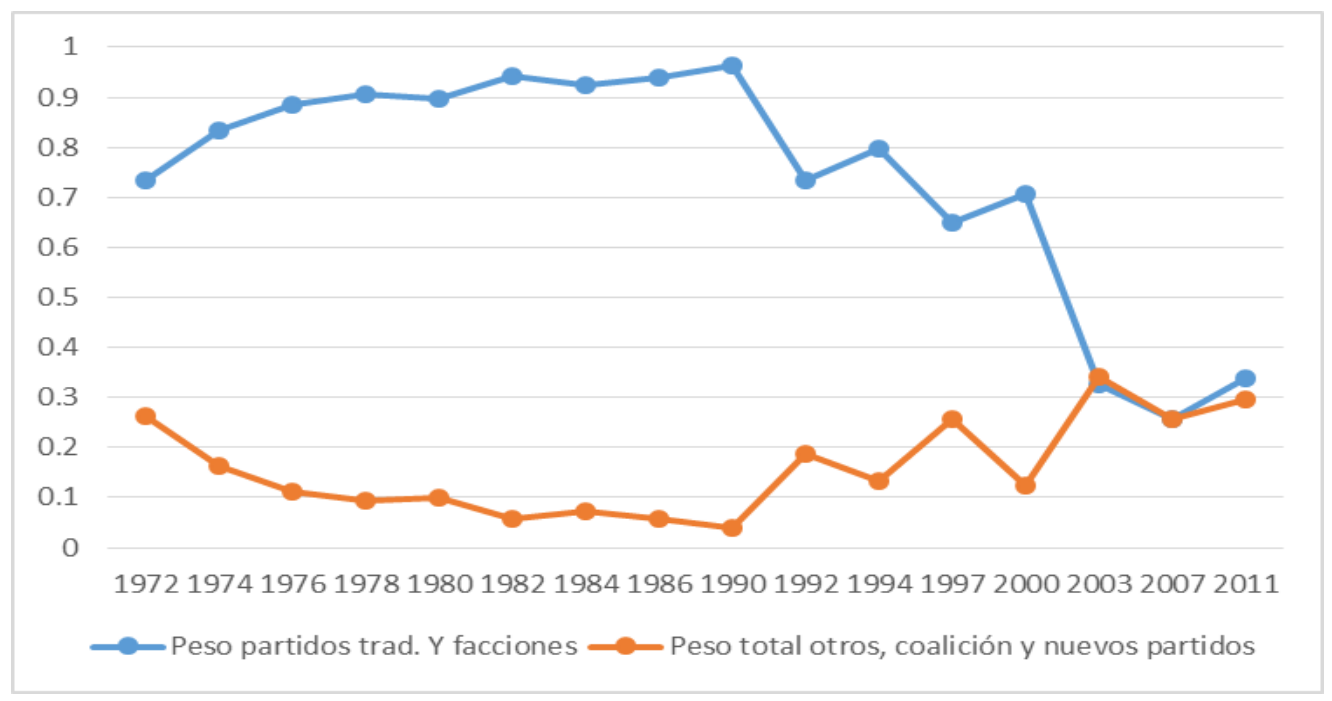

Figura 4. Peso partidos tradicionales versus otros partidos, coaliciones y nuevos. Concejo de Medellín (1972-2011)

Fuente. Construcción propia con datos tomados la Base de Datos Electorales CEDE (Universidad de los Andes) y de la Registraduría Nacional del Estado Civil. 
novedad y el desconocimiento de los términos hacía que la respuesta a la pregunta por el significado de dichos términos fuera por la vía negativa: ser antipolítico, cívico o independiente era todo aquello que no fuera liberal ni conservador, aquello que no podía ser bipartidista. Era evidente que esto decía algo, pero no explicaba satisfactoriamente el fenómeno:

...la idea de renovación se asoció con la desvinculación bipartidista, lo cual supuso dos cosas. En primer lugar, la generalización de un discurso político en el cual lo "independiente" se define de manera negativa. Es decir, "independiente" es todo aquello que no es liberal ni conservador. [...] Sin embargo, desde este momento la "independencia" fue tomando fuerza como una arenga vacía pero no silenciosa que, al tiempo que carece de significado objetivo y no nos dice lo que el político es, se vitaliza cuando los políticos (partidistas o no, nacionales o locales), afirman no ser o no haber salido de la congregación liberal o de la conservadora. (Pizano, 2002, p. 50)

El caso más notorio y sonoro de esa manifestación ha sido sin duda el de Bogotá. La llegada en 1994 de Antanas Mockus a la Alcaldía de la ciudad se convirtió en el fenómeno para ejemplificar el triunfo de los nuevos, del antipolítico, y para argumentar que un nuevo tipo de votante y una nueva forma de hacer política habían hecho su aparición en el país o, al menos, en las grandes ciudades.

Algunos estudios de Bogotá sugieren que el proceso político electoral para Alcaldía, al menos en 1994, privilegió formas antipartido, centradas en el carisma y discurso del candidato (Peña, 1995). Otros insisten en la separación tajante entre el tipo de discurso -antipolítico, antipartidista, hacia la opinión- y las prácticas proselitistas necesarias para ganar la Alcaldía Mayor, por un lado, y las actividades proselitistas necesarias para conquistar las mayorías en el Concejo y en las JAL, por el otro (PNUD, 2008).

El entusiasmo postconstitucional cívico e independiente que ejemplificaba Bogotá dejaba ver, empero, otra contradicción importante del momento. Esta radicaba en que la sociedad y la política parecían transitar por caminos distintos.

Pero nuestra orgía cívica desnudó una tensión entre dos niveles del discurso. En términos de la vida cotidiana, más asociado a lo cívico y a lo urbano entendidos en su forma más convencional, Bogotá aparecía como emblema del atraso y de lo que no debería ser una ciudad. Entre los muchos pecados que se le podían achacar estaban la falta de sentido de pertenencia, el desorden, la incuria, la suciedad, la irracionalidad. En el plano político, en cambio, la capital era y es el símbolo de la independencia de espíritu, del "voto de opinión" (es decir, de aquel que no estaba condicionado por dependencias clientelistas), de la capacidad de cambio. (Gutiérrez, 1995, p. 34)

Es importante señalar que justamente la llegada de Mockus, un antipolítico prototipo casi en todo, excepto en que a diferencia de sus homólogos de ciudades intermedias no denunció el bipartidismo (Gutiérrez, 1996, p. 84), contribuyó a cambiar el significado de la noción de lo cívico, que desde entonces no se asociaría tanto a las ideas de participación y protesta, característica de los años setenta y ochenta, y a que se cerrara significativamente la brecha señalada. Lo cívico se asociaría en adelante a la posibilidad de vivir mejor en comunidad y, por tanto, el discurso y las acciones -sintetizadas en una suerte de giro pedagógico- estaban direccionadas hacia ese fin.

En el caso de Medellín, no se presentó algo similar en la década de los noventa. Lo cívico, que funcionó más como una etiqueta, constituyó el antecedente, en la política local, de la idea del alcaldegerente. Con esta idea, que tuvo mucha difusión en el país desde la primera elección popular de alcaldes (Santana, 1988), se velaba el vínculo partidista con el fin de atraer el apoyo de los más diversos grupos de la sociedad, de otras organizaciones políticas o, incluso, de sectores abiertamente críticos con los partidos tradicionales. Lo cívico expresaba, en parte, la crítica a los políticos por su distanciamiento de los problemas específicos de las localidades y retomaba la imagen de un líder idealizado por su compromiso directo con la comunidad, del buen vecino. Sugería, adicionalmente, un punto intermedio entre la imagen del político y la idea del gerente que se impondría con mayor fuerza con el paso de los procesos electorales de 1990, 1992 y 1994 (Arenas y Escobar, 2000).

Es en el uso de categorías como lo cívico o lo independiente, donde se demuestra la manera en que la vieja política y el clientelismo aprendieron a hacer suya la terminología constitucional, incluso con mayor éxito electoral que aquellos que realmente lo eran y que no tuvieron en Medellín ni los recursos ni la imaginación necesaria para 
posicionar un sello propio.

Habría que esperar hasta la década siguiente para presenciar la aparición en la ciudad de formas políticas nuevas y exitosas, con discursos y prácticas que comenzaron a posicionar una impronta no tradicional (incluso antitradicional), aunque no decididamente antipolítica:

Nosotros, ciudadanos y organizaciones de Medellín, de diversas procedencias, interesados y comprometidos con la construcción y preservación del bien común, preocupados por el creciente deterioro de la gestión pública, por la tendencia al envilecimiento de la actividad política, por la violencia y, en general, por el acelerado retroceso en la calidad de vida, hemos decidido organizarnos en un movimiento político ciudadano, independiente de los partidos tradicionales y de los actores armados, que hemos denominado Compromiso Ciudadano. (Fajardo, citado en Arenas y Escobar, 2012, pp. 111-112)

La consigna programática del movimiento Compromiso Ciudadano tenía una estructura discursiva relativamente sencilla:

1) Declaraba un origen ciudadano, independiente $y$ pluralista; 2) Proponía un diagnóstico, subrayando el envilecimiento de la política y el deterioro de la calidad de vida por la violencia; 3) Identificaba unos agentes responsables: los partidos tradicionales y los actores armados, frente a los cuales el movimiento declaraba su "independencia", es decir, por fuera de las responsabilidades directas de la crisis de la ciudad. Todo esto se vio reforzado a través de la actividad desarrollada por Fajardo, un líder carismático que además supo utilizar su faceta como columnista y figura en diversos medios de comunicación local, lo que potenció su visibilidad. (Arenas y Escobar, 2012, p. 112)

Construir la imagen de independiente no implicaba, en el caso de Fajardo y su movimiento, ubicarse por fuera de la política, sino que sugería la posibilidad de corregir cierto alejamiento de los ciudadanos frente a esta, a causa de su desprestigio. Esto implicaba ensayar una vía distinta a la del antipolítico para enfrentar el malestar de los ciudadanos con sus dirigentes y colocar en el centro la idea de recuperar la dignidad de la política.

Estas características resaltan una de las diferencias entre Fajardo y otros outsider, en la medida que su gobierno no estuvo basado en una oposición con el Concejo, como en la primera etapa del alcalde Mockus en 1994, sino que arrancó con cooperaciones y aprendizajes mutuos, es decir, el alcalde comenzó su gestión reconociendo la importancia de la experiencia acumulada por aquellos políticos locales que llevaban varios períodos en la corporación, y estos a su vez no plantearon una oposición cerrada a las propuestas de la administración, sino que, con expectativas relativamente abiertas, se dejaron contagiar por los principios de eficacia y publicidad de las gestiones (Arenas y Escobar, 2012).

Algunos rasgos permiten establecer, además de las diferencias marcadas, puntos de encuentro entre los procesos de ambas ciudades, centrados sobre todo en el carácter no radical de los dos políticos más reconocidos, lo que establece diferencias importantes con los procesos de líderes de otros países.

\section{Conclusiones}

Cada ciudad ha experimentado, a su manera, el cambio político. En Bogotá el nuevo lenguaje caló prontamente entre buena parte del electorado, sectores importantes de la academia y algunos personajes que venían de allí, de otros sectores de la sociedad o de la política misma, incluso de la tradicional, y que entendieron el "espíritu de la época" o lograron adaptarse a él y generar cambios importantes y sostenidos en la ciudad. El dominio electoral de las opciones no bipartidistas, y claramente de la izquierda desde el año 2003 en la Alcaldía de la capital, parece corroborar la tendencia según la cual, en Bogotá, al menos en el ejecutivo, los tradicionales difícilmente volverían a tener juego.

En Medellín los tradicionales se adaptaron más fácilmente a los nuevos tiempos, conjugaron de mejor forma el nuevo lenguaje, se vistieron con nuevos ropajes y formas que, por supuesto, fueron combinadas con las maneras conocidas de hacer política, y pese a que el cambio llegó tardíamente, mantuvieron altas posibilidades de retorno.

No obstante, cabe la posibilidad de señalar algunas similitudes entre las dos ciudades y sus procesos políticos. La más importante es, sin duda, la existencia tanto en Bogotá como en Medellín, en momentos distintos, de nuevos partidos y políticos que derrotaron la clase política tradicional, con formas diferentes de llegarle al electorado y con líderes políticos que dieron el salto 
a la política nacional y hoy son reconocidos en ella. Adicionalmente, con líderes que coincidian en un estilo fresco y distinto que basaron su accionar en propuestas concretas de planeación y reforma urbana, descuidando probablemente proyectos políticos colectivos de largo aliento.

\section{Referencias}

Arenas, J. y Escobar, J. (2000). Discursos políticos y resultados electorales en Medellín durante los años noventa. Estudios Políticos, 16, 73-99.

Arenas, J. y Escobar, J. (2012). Elecciones, partidos y política local. Medellín: Universidad de Medellín.

Barr, R. (2009). Populists, Outsiders and Anti-establishment Politics. Party Politics, 15, 29-48.

Barreto, A. (2010). El voto de opinión en Bogotá: una mirada crítica. Análisis Político, 23 (69), 66-78.

Boudon, L. (2000). Party System Desinstitucionalization: The 1997-98 Colombian Elections in Historical Perspectives. Journal of Interamerican Studies and world Affairs, 42 (3), 33-57.

García, M. (2002). La política bogotana, un espacio en recomposición (1982-2001). En Gutiérrez, F. (Comp.), Degradación o cambio. Evolución del sistema político colombiano (pp.183-220). Bogotá: Editorial Norma.

Gidengil, E., Blais, A., Nevitte, N. y Nadeau, R. (2001). The Correlates and Consequences of Anti-Partyism in the 1997 Canadian Election. Party Politics, 7(4), 491-513.

Gutiérrez, F. (1995). Tendencias de cambio en el sistema de partidos: el caso de Bogotá. Análisis Político, 24, 106-125.

Gutiérrez, F. (1996). La oposición política en Colombia. Bogotá: IEPRI. Universidad Nacional de Colombia.

Gutiérrez, F. (2006). Estrenando sistema de partidos. Análisis Político, 19 (57), 106-125.

Gutiérrez, F. (2007). ¿Lo que el viento se llevó? Los partidos políticos y la democracia en Colombia. 1958-2002. Bogotá: Grupo Editorial Norma.

Gutiérrez, F. (2017). La destrucción de una República. Bogotá: Taurus.

Guzmán, T. (2005). El deterioro de los partidos como vía de transformación del Concejo de Bogotá entre 1970 y 2000. ¿El proceso de la destrucción creadora? Estudios Políticos, 27, 169-198.

Harmel, R. y Robertson, J. (1985). Formation and Suc- ces of New Parties. A Cross-National Analysis. International Political Science Review, 6 (4), 501-523.

Keren, M. (2000). Political Perfectionism and the Anti-System Party. Party Politics, 6, 107-116.

Kestler, T., Krause, S. y Lucca, J. (2013). Los Break-in parties en América Latina: ¿éxito o fracaso? Revista Debates, 7 (2), 159-171.

Lago, I. y Martínez, F. (2010). Why New Parties? Party Politics, 17, 3-20.

Leal, F. y Zamosc, L. (1990). Al filo del caos: crisis política en la Colombia de los años 80. León Zamosc, Bogotá: Tercer Mundo Editores.

Leal, F. (1988). Democracia oligárquica y rearticulación de la sociedad civil: el caso colombiano. Pensamiento Iberoamericano: revista de economía política, 14, 53-66.

Lynch, N. (1999). Una tragedia sin héroes. Lima: UNMSM. Mainwaring, S. y Scully, T. (1995). Building Democratic Institutions. Party System in Latin America. Stanford: Stanford University Press.

Peña, S. (1995). Rito y símbolo en la campaña para la alcaldía de Bogotá. Análisis Político, 24, 22-35.

Pizano, L. (2002). Reflexiones sobre las decisiones electorales de los bogotanos. Análisis Político, 45, 44-57.

Programa de Naciones Unidas para el Desarrollo. (2008). Bogotá, una apuesta por Colombia. Informe de Desarrollo Humano para Bogotá. Recuperado de http://hdr.undp.org/sites/default/files/ idh_2008_bogota.pdf.

Reina, A. (2008). Compromiso ciudadano: de fenómeno político de opinión a movimiento político. Ecos de las reformas y los resultados electorales (Medellín 2002-2007). Primer Congreso de Ciencia Política. Asociación Colombiana de Ciencia Política. (ACCPOL), Bogotá.

Santana, P. (1988). Los Movimientos cívicos: el nuevo fenómeno electoral. Revista Foro, 6, 47-61.

Sartori, G. (1976). Parties and Party Systems. A Framework for Analyisis. Nueva York: Cambridge University Press.

Torcal, M., Montero, J. y Günther, R. (2007). Los sentimientos antipartidistas en el sur de Europa. En Montero, J., Günther, R. y Linz, J., Partidos Políticos. Viejos conceptos y nuevos retos. Madrid: Editorial Trotta. 